\title{
Olfaction in Olfactory Bulbectomized Rats
}

\author{
Burton Slotnick, ${ }^{1}$ Renee Cockerham, ${ }^{2}$ and Erin Pickett ${ }^{3}$ \\ ${ }^{1}$ Department of Psychology, University of South Florida, Tampa, Florida 33549, ${ }^{2}$ Department of Anatomy and Neurobiology, University of Maryland School \\ of Medicine, Baltimore, Maryland 21201, and 3 Department of Psychology, American University, Washington, DC 20016
}

Experimental rats had their right olfactory bulb removed on postnatal day 2 (P2) and their left olfactory bulb removed on P90. Control rats had one or both olfactory bulbs removed on P90. Before and after their adult-stage surgery, rats were trained using olfactometry and operant conditioning to detect and discriminate odors. Anterograde transport of horseradish peroxidase applied to the olfactory epithelium revealed numerous axons of olfactory sensory neurons in the right hemisphere of 27 experimental rats. These axons terminated in glomerular-like clusters within the frontal neocortex $(n=5)$ or anterior olfactory nucleus with some axons extending into the subventricular epithelium $(n=22)$. Seventeen of the experimental rats were able to detect a variety of odors and to discriminate between odors. Performance accuracy was related to the location and density of these anomalous inputs; experimental rats with inputs confined to frontal neocortex and those lacking any inputs to the forebrain were anosmic, as were adult-operated bilaterally bulbectomized rats. Our results provide strong support for the contention that, in the absence of the olfactory bulbs, olfactory connections to novel forebrain sites can support both odor detection and odor discrimination.

Key words: neural plasticity; olfaction; olfactory bulbectomy; odor detection; neonatal; odor coding

\section{Introduction}

When an olfactory bulb is removed in the neonatal rodent, the forebrain grows forward to fill the vacated cavity. The surgery results in death of mature olfactory sensory neurons (OSNs), but the axons of newly differentiating neurons extend through the cribriform plate and penetrate the overlying forebrain in which at least some make synaptic contact with multipolar cortical cells (Graziadei et al., 1978, 1979). Whether these anomalous inputs to the forebrain can support odor detection has long been a matter of controversy. Attempts to resolve this issue experimentally have yielded mixed results because studies did not adequately assess olfactory capability or did not correlate functional recovery with axonal projections to the forebrain.

An initial claim that adult olfactory bulbectomized mice gradually recovered their ability to smell (Wright and Harding, 1982) was criticized on methodological grounds (Meredith et al., 1983), and a subsequent combined anatomical and behavioral study reported that olfactory forebrain inputs in neonatally bulbectomized rats did not support olfaction (Butler et al., 1984). In that study, rats were unilaterally olfactory bulbectomized on postnatal day 2 (P2), and the remaining olfactory bulb was removed 4 months later. Silver-stained sections of decalcified whole-head mounts demonstrated axonal projections from olfactory epithelium into the rostral forebrain of experimental rats. After their adult-stage surgery, these rats performed at control levels on a nonolfactory task but, despite extensive training, failed on an

Received May 18, 2004; revised July 23, 2004; accepted July 26, 2004

This work was supported in part by National Science Foundation Grant IBN-0080746 (B.S.). We thank Elke Weiler and Kathleen Guthrie for their many helpful comments and Kathleen McBride for her technical assistance.

Correspondence should be addressed to Burton Slotnick, Department of Psychology, University of South Florida, PCD 4118G, 4202 Fowler Avenue, Tampa, FL 33620. E-mail: slotnic@american.edu.

D0I:10.1523/JNEUROSCI.1936-04.2004

Copyright $\odot 2004$ Society for Neuroscience $\quad$ 0270-6474/04/249195-06\$15.00/0 amyl acetate detection task. In a related study, but one using an indirect measure of olfaction (thermotaxis), Small and Leonard (1983) also concluded that anomalous projections of OSNs to the forebrain in neonatal bulbectomized hamsters did not support olfaction.

However, in a partial replication of the study by Butler et al. (1984), Hendricks et al. (1994) provided some evidence that such inputs to the forebrain could support olfaction. Hendricks et al. (1994) stained for olfactory marker protein to confirm the olfactory origin of these inputs but used a relatively crude foodfinding task as a measure of odor detection. In contrast to the results of Butler et al. (1984), they found that each of six experimental rats performed the task better than adult olfactory bulbectomized rats, albeit more slowly than rats that had one intact olfactory bulb. Hendricks et al. (1994) concluded that anomalous projections from the olfactory epithelium to the forebrain could support odor detection and that the failure of the study by Butler et al. (1984) stemmed from a potential specific anosmia to amyl acetate or from the use of an operant task whose demands exceeded the behavioral abilities of experimental rats.

Given the relevance of this issue for understanding the extent of neural plasticity in the olfactory system and the role of the olfactory bulb in mediating olfactory function, we have reevaluated the ability of bilaterally olfactory bulbectomized rats to detect and discriminate odors. We used precision olfactometry to assess behavior and anterograde axonal transport of wheat germ agglutinin conjugated to horseradish peroxidase (WGA-HRP) to evaluate projections from the olfactory epithelium to the forebrain.

\section{Materials and Methods}

Subjects. Sixty-two neonatal rats of both sexes born to Sprague Dawley females were used. Pregnant animals were housed individually, and 
date of birth constituted P1 for pups. Pups were housed with their dam until P21 when they were housed in same-sex groups of two to three. All procedures were performed according to a protocol approved by the American University Institutional Animal Care and Use Committee.

Surgery. On P2 or P3, pups were anesthetized by cold narcosis. The scalp was incised, and the nasal bone over the right olfactory bulb was removed. Most or all of the exposed olfactory bulb was aspirated using a fine glass pipette in 50 pups (experimental rats). The exposed bulb was left undamaged in the remaining 12 pups. The scalp was closed with tissue cement, and the pup recovered in a humid $35^{\circ} \mathrm{C}$ cage and was reunited with its dam 12-18 hr later. After initial training on P70-P80, rats were anesthetized with ketamine hydrochloride and xylazine and clamped into the head holder of a stereotaxic instrument. The left olfactory bulb was exposed and removed by aspiration in each experimental rat and in eight controls. Both olfactory bulbs were removed in the remaining four controls. Rats recovered on a warming pad and were then returned to their home cage.

Anatomical control. At termination of all behavior testing, each rat was lightly anesthetized, and both nasal vaults were syringed with $40 \mu \mathrm{l}$ of an equal-parts mixture of 1\% WGA-HRP (Sigma, St. Louis, MO) and 1\% dimethylsulfoxide. Rats were deeply anesthetized and killed $24 \mathrm{hr}$ later by perfusion through the heart with saline followed by mixed aldehydes. The forebrain was frozen and sectioned at $50 \mu \mathrm{m}$, and every other section was reacted with tetramethylbenzidine (Mesulam, 1982). Sections were lightly counterstained with thionin, inspected microscopically, and photographed using a Nikon (Tokyo, Japan) digital camera.

Behavior tests. Beginning on P70, rats were trained using operant conditioning in a computer-controlled eight-channel olfactometer, as described previously (Slotnick and Schellinck, 2002). Briefly, odor stimuli were generated by a $40 \times$ dilution with clean air of a $50 \mathrm{ml} / \mathrm{min}$ sample from the headspace of an odorant diluted $\mathrm{v} / \mathrm{v}$ with mineral oil. The headspace concentration of several of our odorants (ethyl acetate, acetic acid, and propionic acid) can be calculated from data provided by ComettoMuniz et al. (2003). Because the physical concentration of the odorant headspace for other odorants we used is not known, odor concentration is stated as the percentage liquid dilution of the odorant.

A go, no-go discrimination procedure (Slotnick and Schellinck, 2002) was used in which $23.75 \mathrm{hr}$ water-deprived rats were trained to sample the odor stimulus and to lick a water delivery tube when in the presence of the odor designated as positive $(\mathrm{S}+)$ but to inhibit responding when in the presence of the odor designated as negative $(\mathrm{S}-)$. Responding correctly on $S+$ trials (hits) was reinforced with $0.04 \mathrm{ml}$ of water. Responding on $\mathrm{S}-$ trials (false alarms) was punished by a $15 \mathrm{sec}$ extension of the 5 sec intertrial interval. Not responding on $S+$ trials was scored as misses, whereas not responding on $\mathrm{S}-$ trials was scored as correct rejections. Misses and false alarms constituted errors, and the percentage correct responding was calculated for each block of 20 trials.

Beginning on P70, rats were trained to detect the odor from 5, 1, 0.1, and $0.01 \%$ concentrations of ethyl acetate and $1 \%$ jasmine [a Givaudan (New York, NY) perfume odorant]. Rats were given 200 trials on each task; the odorant solution served as the $\mathrm{S}+$ stimulus, and the solvent served as the $\mathrm{S}-$ stimulus.

Beginning $21 \mathrm{~d}$ after their adult-stage surgery, rats were retested on these tasks and on novel odor detection and discrimination tasks. A more restricted number of problems were used in an initial group of rats, and thus, not all rats were tested on all tasks (see Table 1 ). S + odorants for the novel detection tasks were 10 and $1 \%$ acetic acid, $1 \%$ citral, $1 \%$ jasmine, and $5 \%(+)$-carvone. The mineral oil solvent served as the $S-$ stimulus. Stimuli for discrimination tasks were $1 \%$ jasmine perfume $(\mathrm{S}+)$ versus $1 \%$ rose perfume $(\mathrm{S}-), 1 \%$ acetic acid $(\mathrm{S}+)$ versus $1 \%$ caproic acid $(\mathrm{S}-)$, $1 \%$ acetic acid $(\mathrm{S}+)$ versus $1 \%$ propionic acid $(\mathrm{S}-)$, and $5 \%(+)$-carvone $(\mathrm{S}+)$ versus $5 \%(-)$-carvone $(\mathrm{S}-)$. Ethyl acetate, citral, and acetic, propionic, and caproic acids were purchased from Sigma, and the carvone enantiomers were purchased from Fisher Scientific-Acros Organics (Geel, Belgium). The purity levels were the highest available from these suppliers. Perfume odorants were kindly supplied by Givaudan. Rats
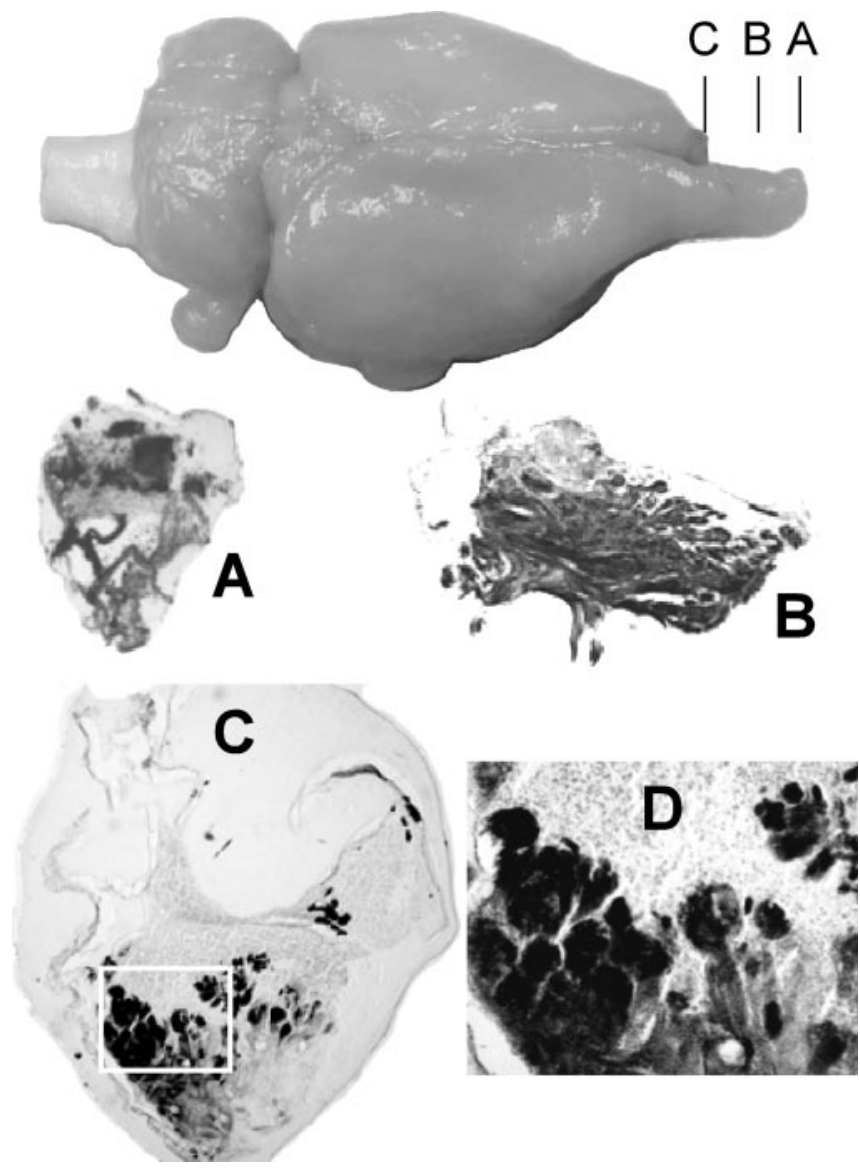

Figure 1. Top, Photograph showing the extended forebrain on the right (neonatally operated) hemisphere. The forebrain extension filled the cavity vacated by the neonatal bulbectomy. Frontal levels $A, B$, and $C$ are approximately those illustrated in photomicrographs $A-C$. $A-C$, Photomicrographs of sections through the anterior $(A)$ and middle $(B)$ portion of the forebrain extension and through the rostral anterior olfactory nucleus ( () . As shown, the extended forebrain tissue is heavily invested with WGA-HRP reaction product, and numerous glomerular-like clusters can be identified within the anterior olfactory nucleus. D, Highermagnification photomicrograph of region outlined in ( showing details of glomerular-like clusters of axons within the anterior olfactory nucleus.

were trained on each task until they achieved criterion performance of $85 \%$ correct responding in a block of 20 trials or for a maximum of 200 trials.

\section{Results}

\section{Anatomical results}

The left olfactory bulb (adult-stage surgery) was completely removed in each experimental and control rat. Anatomical results from 27 of 50 experimental rats met the criteria that both olfactory bulbs had been completely removed, that the right hemisphere had grown forward to fill or partly fill the cavity vacated by the neonatal bulbectomy (Fig. 1), and that the rostral forebrain contained WGA-HRP reaction product. These 27 rats were designated group NBXi (for neonatally operated bilateral bulbectomized rats with forebrain input). During removal of the forebrain from the skull in these rats, we observed numerous bundles of axons that extended through the cribriform plate and into the tissue extension in the right hemisphere. In 5 of these 27 rats, the lesions in the neonatal operated hemisphere invaded the rostral third of the anterior olfactory nucleus (AON) but produced little or no damage to frontal pole cortex, and the forebrain extension consisted only of frontal neocortex. In the other 22 rats, the le- 

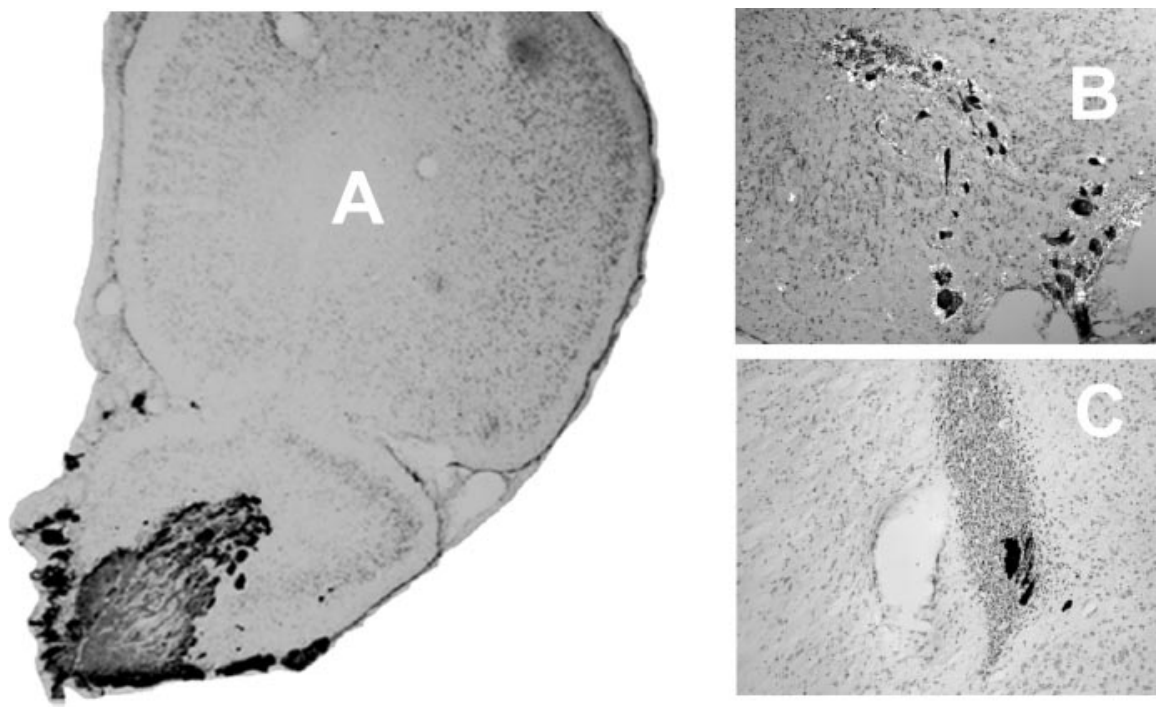

Figure 2. A, Photomicrograph of a section through the frontal cortex and posterior aspect of the anterior olfactory nucleus in another experimental rat. The dense WGA-HRP reaction product in the AON is representative of that found in most NBXi rats that could detect and discriminate odors. $B$, Photomicrograph (using polarized light optics) showing a stream of fibers directed toward ependyma juxtaposed to the anterior limb of the anterior commissure and fibers entering this zone $(C)$.

form layer. These rats with identifiable olfactory bulb tissue were designated the Rem group. The six rats in this group with the smallest bulb remnants also had olfactory projections that extended beyond the olfactory bulb to penetrate into the AON. Olfactory projections from OSNs in the other rats of this group were confined to the olfactory bulb remnant.

The lesions in the right hemisphere of the remaining 13 experimental rats were quite large and removed the entire olfactory peduncle and rostral 3-4 mm of frontal cortex. The forebrain of these rats did not extend toward the cribriform plate and contained no discernable labeled olfactory axons. These rats were designated group NBXn (for neonatally operated bilateral bulbectomized rats that had no olfactory input to the forebrain).

The right olfactory bulb of each of the eight unilaterally bulbectomized control rats was intact, and the left bulb was completely removed. Both olfactory bulbs were completely removed in the four adult-stage bilat-

sions invaded frontal neocortex and only the medial aspect of the AON. The forebrain tissue in contact with the cribriform plate in these cases consisted of an extension of the olfactory peduncle. Histologically, the ventral aspect of this extended forebrain tissue contained, in different rats, sparse to dense WGA-HRP reaction product demonstrating both thick and fine bundles of axons that either terminated in glomerular-like clusters within the tissue extension or, in some cases, extended back to terminate within the AON rostral to the level of the olfactory tubercle (Figs. 1, 2). In most of the later cases, a subset of axons appeared directed toward the subventricular epithelial zone surrounding the base of the most rostral aspect of the lateral ventricle or, more commonly, to that epithelium juxtaposed to the anterior limb of the anterior commissure (Fig. $2 \mathrm{~A}$ ). In several cases, axons were observed to penetrate and appeared to terminate within this zone (Fig. $2 B$ ).

Within the AON, axonal bundles terminated in numerous small $(10-20 \mu \mathrm{m})$ but fewer medium $(30-70 \mu \mathrm{m})$ or larger-sized $(40-150 \mu \mathrm{m})$ glomerular-like clusters on the tissue surface or deep within the parenchyma. The mean diameter of 155 glomerular clusters measured in 10 rats $(47.2 \mu \mathrm{m}$; range, $10-150 \mu \mathrm{m})$ was significantly $(p<0.001)$ smaller than the mean of 80 olfactory bulb glomeruli measured in six control rats $(81.6 \mu \mathrm{m}$; range, 40-155 $\mu \mathrm{m})$. Most experimental rats had small, medium, and large glomerular clusters, and there was no obvious relationship between the size of glomerular clusters and performance on the behavior tasks. The invading axons did not induce a bulbar-like structure in the tissue; no bulb-like lamination was apparent, and we did not observe periglomerular-like cells surrounding axonal clusters. Approximately 430 such clusters were counted in the rat with the richest input (Fig. $2 A$ ), and $\sim 30$ clusters were observed in the rat with the sparsest input.

In the remaining 23 experimental rats, the brains of 10 rats contained a damaged fragment of olfactory bulb in the right hemisphere. This olfactory bulb tissue was identified in 6-14 sections ( $0.6-1.4 \mathrm{~mm}$ of tissue in the anteroposterior plane), and, in each case, the tissue was richly invested with labeled axons that terminated in glomeruli and penetrated into the external plexi- erally olfactory bulbectomized controls. No WGA-HRP reaction product was detected in the left (adult-operated) hemisphere in any rat.

\section{Behavioral results}

Of the 27 NBXi rats that met our anatomical criteria, the five with connections confined to frontal pole cortex and 5 of the 22 rats with connections to the olfactory peduncle failed on each of the odor detection tasks and were judged as anosmic. The remaining 17 rats were able to detect odors, and each of these was able to discriminate between at least one pair of odors (Fig. 3). The olfactory ability of these rats varied widely; some failed on several detection or discrimination tasks or performed poorly over many trials but gradually improved and achieved or exceeded the criterion of $85 \%$ accuracy in a block of 20 trials, whereas others performed nearly as well as controls (Fig. 3). Each of these 17 rats achieved criterion performance on the 5 and $1 \%$ ethyl acetate detection tasks, and 15 (88\%) detected the vapor from $0.1 \%$ ethyl acetate $(218 \mathrm{ppm})$, a concentration that is $\sim 45$ times lower than human trigeminal threshold for the odorant (Jalowayski et al., $2001)$. Eleven $(65 \%)$ of these rats were able to detect the vapor from $0.01 \%$ ethyl acetate. Of those tested, most were able to detect the perfume, citral, $(+)$-carvone, and acetic acid odorants as well as being able to discriminate between perfume oils, the enantiomers of carvone, acetic from caproic acid, and acetic from propionic acid (Table 1). The three rats that performed best had particularly heavy olfactory nerve input to the anterior olfactory nucleus. On average, the performance accuracy of NBXi rats was consistently lower than those with residual bulb tissue and than controls rats that had one intact olfactory bulb (Fig. 3). Most rats in the Rem group performed almost as well as controls on all tasks.

Each control rat that was bilaterally bulbectomized as an adult and each NBXn rat (experimental rats that did not meet our anatomical criteria) failed on each of the odor detection tasks. Accuracy scores of these rats on all tasks ranged between 40 and $65 \%$. 


\section{Discussion}

Our results demonstrate that most rats lacking all olfactory bulb tissue but having anomalous projections from OSNs to rostral olfactory forebrain structures were able to detect the vapor from several different odorants and to discriminate between odorants. These results are in accord with and extend those of Hendricks et al. (1994). Our evidence that these rats were completely olfactory bulbectomized is that no tissue characteristic of the olfactory bulb was observed in tissue sections and that, in each case, the neonatal lesions extended into the anterior olfactory nucleus at a level that was at least $1 \mathrm{~mm}$ posterior to the most posterior extent of the olfactory bulb. Small remnants of olfactory bulb tissue present in rats of the Rem group were easily identified but were completely absent in NBXi rats. Our evidence that odor detection and discrimination in nosmic NBXi rats was mediated by anomalous axonal projections to olfactory forebrain structures is less direct. However, the density of this input was related to the rats' ability to detect and discriminate odors, and NBXi rats with input confined to tissue above the rhinal fissure and those lacking any input (NBXn rats) were frankly anosmic. This outcome and the facts that nosmic NBXi rats were able to detect ethyl acetate vapor at concentrations well below the trigeminal threshold for this odor, that they were able to detect odorants that are clearly not trigeminal stimuli (dilute perfume odorants and dilute enantiomers of carvone), and that they were able to discriminate between odors argue strongly against the possibility that nonolfactory cues or other chemosensory systems mediated this performance.

The olfactory tasks used in this study were relatively easy. Except on the carvone discrimination, control rats achieved criterion performance in the first or second block of trials in each task, and rats with only a small remnant of their olfactory bulb performed almost as well. In contrast, on average, nosmic NBXi rats performed consistently more poorly, and some of these rats failed to achieve criterion on one or more tasks. Thus, the functional capacity of the anomalous input to the forebrain is limited and does not rival even that of rats with small remnants of olfactory bulb. However, it is noteworthy that several NBXi rats with particularly heavy input to the forebrain performed nearly as well as controls in several of the detection and discrimination problems.

In contrast to the present results, Butler et al. (1984) reported that reconstituted olfactory projections to the forebrain did not support olfaction. Their negative results were probably because reconstituted olfactory projections in most of their experimental rats terminated in frontal pole cortex; only five rats had input to the anterior olfactory nucleus, and most of those had not been trained on the odor detection task before their adult-stage surgery.
Mean Scores: Discrimination

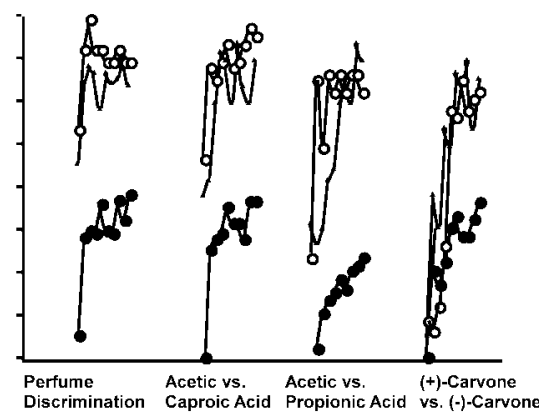

Individual Rats: Discrimination
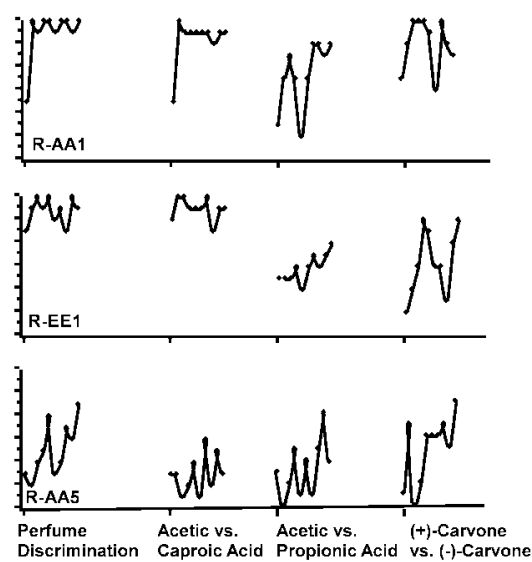

Figure 3. Mean performance of nosmic NBXi rats, control rats, and rats with olfactory bulb remnants on odor detection tasks asmine perfume, citral, $10 \%$ and $1 \%$ acetic acid, and (+)-carvone, respectively (see Materials and Methods). Each data point is the mean score on a block of 20 trials. The bottom graphs show performance of the two NBXi rats that performed best (rats AA1 and EE1) and one rat (AA5) representative of those that performed more poorly on the odor detection and discrimination tasks.

Although recent studies have shown considerable capacity for anatomical and functional reorganization of brain circuitry (Frost et al., 2000; von Melchner et al., 2000; Ptito et al., 2001) and that transplanted neural progenitor cells can adopt phenotypic characteristics of the area into which they have been transplanted (Fujii and Hayakashi, 1995; Fricker et al., 1999), our results, together with those of Hendricks et al. (1994), are perhaps the first to demonstrate and characterize function after removal of the first synaptic relay of a cranial sensory system. We hypothesize that olfactory function in our nosmic NBXi rats was likely mediated by synaptic contacts of sensory axons with neurons in the anterior olfactory nucleus, the site of termination for essentially all of these anomalous inputs. Evidence from other studies suggests that these anomalous inputs induce some reorganization within the forebrain. Thus, sensory axon terminals form synaptic contacts on nearby multipolar neurons that extend their dendrites into these glomerular-like structures (Graziadei et al., 1978; Graziadei and Monti Graziadei, 1986), and at least some of these clusters have been observed to be associated with periglomerular-like tyrosine hydroxylase $(\mathrm{TH})$-positive neurons (Guthrie and Leon, 1989). Our tissue sections, processed for WGA-HRP and lightly counterstained with thionin, were inadequate to reveal such changes.

In a related study, Guthrie and Leon (1989) reported that anomalous projections into the forebrain from the olfactory epithelium of neonatal bulbectomized rats appeared to induce expression of the catecholamine biosynthetic enzyme $\mathrm{TH}$ in neu- 
Table 1. Total errors in a 200 trial session or errors to criterion of $85 \%$ correct responding for each of the experimental rats that could detect odors and mean scores for controls on each odor detection and discrimination task

\begin{tabular}{|c|c|c|c|c|c|c|c|c|c|c|c|c|c|}
\hline Rat number & EA $5 \%^{a}$ & EA 1\% & EA $0.1 \%$ & EA 0.01\% & $A$ & B & $C$ & D & $E$ & D1 & $\mathrm{D} 2$ & D3 & D4 \\
\hline AA1 & 14 & 0 & 3 & 2 & 3 & 2 & 1 & 9 & 7 & 7 & 7 & 17 & 27 \\
\hline AA2 & 14 & 2 & 3 & 7 & 6 & 8 & 1 & 1 & 46 & 10 & 15 & $76^{* *}$ & 46 \\
\hline AA5 & 117 & 71 & $97^{* *}$ & $100 * *$ & $93^{* *}$ & $90^{* *}$ & 45 & 8 & 64 & 69 & $93^{* *}$ & $72^{* *}$ & 64 \\
\hline AA6 & 9 & 8 & $99^{* *}$ & $101^{* *}$ & $94^{* *}$ & $93^{* *}$ & 56 & $95^{* *}$ & 79 & $96^{* *}$ & $98^{* *}$ & $82^{* *}$ & 72 \\
\hline EE1 & 17 & 1 & 5 & 5 & 5 & 40 & 2 & 3 & 26 & 3 & 2 & $91^{* *}$ & 56 \\
\hline EE2 & 18 & 7 & 14 & 28 & 6 & $79^{* *}$ & 10 & 18 & 15 & $97^{* *}$ & 10 & $107^{* *}$ & 25 \\
\hline EE4 & 56 & 10 & 19 & $105^{* *}$ & $93^{* *}$ & $103^{* *}$ & $91^{* *}$ & 53 & 7 & 29 & $92^{* *}$ & 33 & 43 \\
\hline EE5 & 16 & 6 & 22 & 6 & 3 & 7 & 7 & 43 & $86^{* *}$ & 9 & 20 & 25 & $86^{* *}$ \\
\hline A16 & 111 & 3 & 12 & 15 & 75 & * & 27 & * & * & $89^{* *}$ & * & $107^{* *}$ & * \\
\hline A27 & 2 & 16 & 10 & 9 & 17 & * & 22 & * & * & 14 & $*$ & 59 & * \\
\hline D5 & 139 & 7 & 32 & 19 & 14 & * & $97^{* *}$ & * & $88^{* *}$ & $103^{* *}$ & * & $94^{* *}$ & $98^{* *}$ \\
\hline D9 & 6 & 3 & 1 & 10 & 5 & * & 15 & * & 56 & 12 & * & 33 & $93^{* *}$ \\
\hline D15 & 154 & 5 & 42 & 43 & 8 & * & 34 & * & 34 & $92^{* *}$ & * & $94^{* *}$ & 39 \\
\hline D24 & 3 & 1 & 0 & $63^{* *}$ & 1 & * & 14 & * & 42 & 12 & * & 11 & 31 \\
\hline D27 & 201 & 3 & 22 & $98^{* *}$ & 19 & * & 33 & * & 23 & 21 & * & * & $100^{* *}$ \\
\hline E6 & 4 & 2 & $91^{* *}$ & $98^{* *}$ & 1 & * & 12 & & 12 & 2 & * & 13 & 16 \\
\hline E7 & 19 & 0 & 1 & 2 & 12 & $*$ & 43 & * & 17 & 23 & $*$ & $93^{* *}$ & 17 \\
\hline Percentage $^{b}$ & 100 & 100 & 82 & 65 & 82 & 50 & 88 & 88 & 87 & 70 & 62 & 44 & 73 \\
\hline Controls ${ }^{c}$ & 4.2 & 6.1 & 13.5 & 8.6 & 3.4 & 5.3 & 2.7 & 3.7 & 5.1 & 2.6 & 6.2 & 9.3 & 31.7 \\
\hline
\end{tabular}

${ }^{a}$ A maximum of 600 trials was allowed on the $5 \%$ ethyl acetate detection, 200 trials on all other tasks. EA, Ethyl acetate.

${ }^{b}$ Percentage of rats tested that reached criterion performance.

'Mean errors. All control rats reached criterion on all tasks. A-E, Perfume, citral, 10\% acetic acid, 1\% acetic acid, and (+)-carvone detection tasks, respectively. D1-D4, Perfume discrimination, acetic acid versus caproic acid, acetic acid versus propionic acid, and (+)-carvone versus ( - )-carvone discrimination tasks, respectively.

*Was not tested.

**Did not reach criterion of $85 \%$ correct responding.

rons found along the borders of glomerular-like clusters of olfactory axons. These neurons appeared to derive from the rostral migratory stream of the forebrain subventricular zone (SVZ). As shown in explant studies (Liu and Rao, 2003), activity of sensory axons may have directed their migration from the SVZ and, as in the normal olfactory bulb, induced the expression of $\mathrm{TH}$ (Baker et al., 1993). These observations, together with the present results, indicate that, in the absence of the olfactory bulb, the invasion of first-order olfactory axons into the brain induces changes in neural organization that can support olfaction. These changes have not been further characterized, but they raise a host of intriguing questions. These include the following: identifying the projection sites for forebrain neurons that assume the function of second-order olfactory neurons; determining what, if any, reorganization may occur in the massive centrifugal projections that normally terminate in the olfactory bulb; and why some of our NBXi rats with apparently adequate connections were unable to detect odors. Other issues raised by the ability of olfactory bulbectomized rats to detect and discriminate odors bear on the role of the olfactory bulb in processing odor inputs. In the normal olfactory system, axons of neurons that express the same odor receptor protein converge and terminate in one or a few discrete olfactory bulb glomeruli, but the factors governing this convergence are not well understood (St John et al., 2002, 2003; Ranganathan and Buck, 2002). The neonatal bulbectomized preparation could provide a useful tool to help resolve the extent to which this convergence in axonal termination is dependent on guidance signals from the olfactory bulb. Additionally, this preparation could elucidate the extent to which odor detection, discrimination, and odor quality perception are dependent on the patterns of projections to the olfactory bulb and on local circuit organization within the bulb.

\section{References}

Baker H, Morel K, Stone DM, Maruniak JA (1993) Adult naris closure profoundly reduces tyrosine hydroxylase expression in mouse olfactory bulb. Brain Res 614:109-116.

Butler AB, Graziadei PP, Monti Graziadei GA, Slotnick BM (1984) Neonatally bulbectomized rats with new olfactory-neocortical connections are anosmic. Neurosci Lett 48:247-254.

Cometto-Muniz JE, Cain WS, Abraham MH (2003) Quantification of chemical vapors in chemosensory research. Chem Senses 28:467-477.

Fricker RA, Carpenter MK, Winkler C, Greco C, Gates MA, Björklund A (1999) Site-specific migration and neuronal differentiation of human neural progenitor cells after transplantation in the adult rat brain. J Neurosci 19:5990-6005.

Frost DO, Boire D, Gingras G, Ptito M (2000) Surgically created neural pathways mediate visual pattern discrimination. Proc Natl Acad Sci USA 97:11068-11073.

Fujii M, Hayakashi T (1995) Axons from the olfactory bulb transplanted into the hippocampal formation show axon preferences and form transplant-to-host synapses similar to those of normal afferents. Neurosci Res 24:53-60.

Graziadei PP, Monti Graziadei GA (1986) Neuronal changes in the forebrain of mice following penetration by regenerating olfactory axons. J Comp Neurol 247:344-356.

Graziadei PP, Levine RR, Monti Graziadei GA (1978) Regeneration of olfactory axons and synapse formation in the forebrain after bulbectomy in neonatal mice. Proc Natl Acad Sci USA 75:5230-5234.

Graziadei PP, Levine RR, Monti Graziadei GA (1979) Plasticity of connections of the olfactory sensory neuron: regeneration into the forebrain following bulbectomy in the neonatal mouse. Neuroscience 4:713-727.

Guthrie KM, Leon M (1989) Induction of tyrosine hydroxylase expression in rat forebrain neurons. Brain Res 497:117-131.

Hendricks KR, Knott JN, Lee ME, Gooden MD, Evers SM, Westrum LE (1994) Recovery of olfactory behavior. I. Recovery after a complete olfactory bulb lesion correlates with patterns of olfactory nerve penetration. Brain Res 648:121-133.

Jalowayski AA, Johnson BN, Wise PM, Schmid-Schönbein GW, Cain WS (2001) Orbital response indicates nasal pungency: analysis of biomechanical strain on the skin. Chem Senses 26:1005-1013. 
Liu G, Rao Y (2003) Neuronal migration from the forebrain to the olfactory bulb requires a new attractant persistent in the olfactory bulb. J Neurosci 23:6651-6659.

Meredith M, Graziadei PP, Monti Graziadei GA, Rashotte ME, Smith JC (1983) Olfactory function after bulbectomy. Science 222:1254-1255.

Mesulam M (1982) Tracing neural connections with horseradish peroxidase. New York: Wiley.

Ptito M, Giguere JF, Boire D, Frost DO, Casanova C (2001) When the auditory cortex turns visual. Prog Brain Res 134:447-458.

Ranganathan R, Buck LB (2002) Olfactory axon pathfinding: who is the pied piper? Neuron 35:599-600.

Slotnick B, Schellinck H (2002) Methods in olfactory research with rodents. In: Frontiers and methods in chemosenses (Simon SA, Nicolelis M, eds), pp 21-61. Boca Raton, FL: CRC.
Small RK, Leonard CM (1983) Rapid fiber reorganization after early olfactory tract section and bulbectomy in the hamster. J Comp Neurol 214:353-369.

St John JA, Clarris HJ, Key B (2002) Multiple axon guidance cues establish the olfactory topographic map: how do these cues interact? Int J Dev Biol 46:639-647.

St John JA, Clarris HJ, McKeown S, Royal S, Key B (2003) Sorting and convergence of primary olfactory axons are independent of the olfactory bulb. J Comp Neurol 464:131-140.

von Melchner L, Pallas SL, Sur M (2000) Visual behaviour mediated by retinal projections directed to the auditory pathway. Nature 404:871-876.

Wright JW, Harding JW (1982) Recovery of olfactory function after bilateral bulbectomy. Science 216:322-323. 